\title{
Planar Polarimetry Receivers for Large Imaging Arrays at Q-band
}

\author{
Pekka Kangaslahti, Todd Gaier, Michael Seiffert, Sander Weinreb, Dennis Harding, Douglas Dawson, Mary \\ Soria, Charles Lawrence, Benjamin Hooberman* and Amber Miller*
}

Jet Propulsion Laboratory, Pasadena, CA, 91109, USA *Columbia University, New York, NY, 10027, USA

\begin{abstract}
The characterization of the intensity fluctuations of the Cosmic Microwave Background (CMB) will be followed by the mapping of the polarization fluctuations of the CMB. Measurement of the polarization fluctuations requires highly sensitive instruments that are only possible by increasing the number of receivers. We are developing a large receiver array for the Q, U Imaging Experiment (QUIET) by building individual receivers that have noise temperatures close to the physical limit and that are simple, and low cost to build and operate. We developed these planar polarimetry receivers for Q-band by designing InP MMIC amplifiers with noise below $20 \mathrm{~K}$, low loss and highly balanced phase switches and an entirely planar hybrid thin film circuit for the detection of the Stokes parameters $Q$ and $U$. Our receivers achieve $25 \mathrm{~K}$ noise temperature over 8 GHz bandwidth and provide the $I, Q$ and $U$ parameters simultaneously. These planar modules have a simple plug in architecture that enables automated production of a large number of receivers and simple integration of large arrays of receivers.
\end{abstract}

Index Terms - Polarimetry, Millimeter wave radiometry, Millimeter wave phase shifters, MMICs, Millimeter wave imaging, Cosmic Microwave Background (CMB).

\section{INTRODUCTION}

For the last 40 years the Cosmic Microwave Background (CMB) has been the most important source of information about the geometry and contents of the universe. Several ground based and space instruments exist or are in development to map the intensity fluctuations of $\mathrm{CMB}$. The imaging of polarization fluctuations, however, has only started within the last few years [1]. The origin of CMB polarization is the Thomson scattering of quadrupole anisotropies in the surface of last scattering. The $\mathrm{CMB}$ contains E-mode polarization that results from density perturbations and Bmode polarization that is the outcome of vector or tensor perturbations or higher order effects such as gravitational lensing. The E-mode fluctuations are a few percent of the level of $\mathrm{CMB}$ intensity fluctuations and thus require highly sensitive receivers. The B-mode fluctuations from lensing are smaller still, and the B-mode fluctuations from gravitational waves are likely the even smaller. The current experiments have successfully measured E-mode fluctuations, however, to increase the accuracy of E-mode measurements and facilitate B-mode measurements the sensitivity of the receivers has to be significantly improved.

The recent development in Indium Phosphide PHEMT technology has resulted in Low Noise Amplifier based receivers that are approaching the fundamental physical limits to noise. The only feasible way to increase the sensitivity is to increase the number of receivers. To enable low cost production of a large number of receivers, the assembly and testing of the polarimeters has to be simple, which is most easily achieved with completely planar, InP MMIC based Multichip Modules (MCM).

We are currently developing a large focal plane array of HEMT radiometers for $\mathrm{CMB}$ radiation polarization at 40 and $90 \mathrm{GHz}$ [2]-[4]. The array at $40 \mathrm{GHz}$ was particularly important for the removal of foreground contamination. At 40 $\mathrm{GHz}$, HEMT amplifiers offer the lowest noise of any continuum receiver detector; thus, we were able to develop fully functional polarimeter modules with less than $25 \mathrm{~K}$ noise temperature and $8 \mathrm{GHz}$ bandwidth. The development of the array elements proceeded by design and cryogenic testing of the LNA MMICs, design and testing of MMIC phase switches, design and testing of thin film circuits and, finally, design and manufacturing of the planar multichip module (MCM) package. The block diagram of the polarimeter is shown in Fig 1.

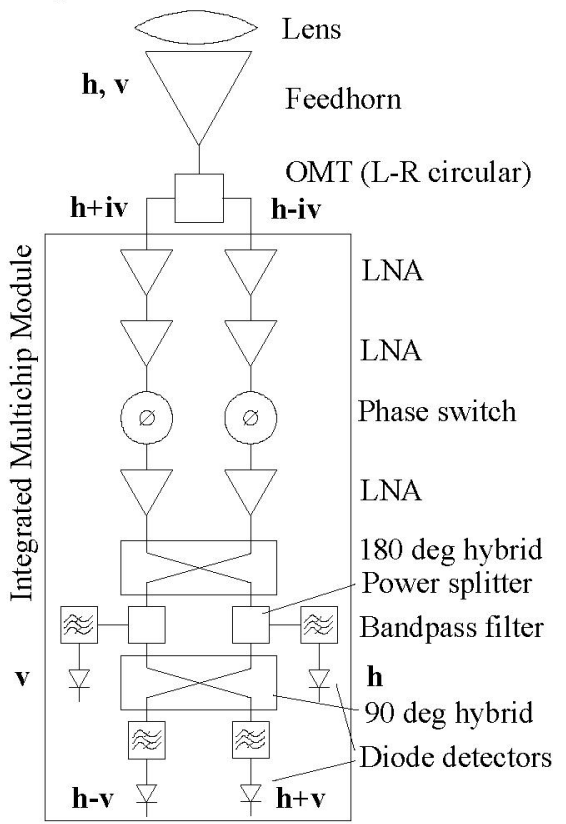

Fig. 1. Block diagram of the $\mathrm{Q} / \mathrm{U}$ detecting polarimeter. The OMT splits the signal from the antenna into $h+i v$ and h-iv components ( $h$ refers to the horizontal component of the radiation and $\mathrm{v}$ to the vertical component). The amplified signals are fed into the phase discriminator hybrid that has four outputs that are detected. These detected signals are used to calculate the Stokes parameters I, Q and $\mathrm{U}$. 


\section{MMIC AND Thin-Film CiRcuit DeVElopMENT}

The compact planar design of the MCM was achieved by implementing the functionality of the polarimeter on MMIC and thin-film circuits. The identified circuits in Fig. 1 include LNA MMICs, phase switch MMICs, 180 degree hybrid, 90 degree hybrid and band pass filters, detectors and power splitters. The amplifiers provide the low noise amplification to sufficient level for the detectors to detect the band pass filtered CMB signal. The phase switches improve the stability of the system by enabling us to switch the outputs between the detectors. Finally, the combination of the 180 degree hybrid and 90 degree hybrid with intermediate power splitters provide the polarization detection capability.

\section{A. Low noise amplifier MMICs}

Low noise MMIC amplifiers were developed using an Indium Phosphide foundry process with $0.1 \mu \mathrm{m}$ gate length. The process has typical $\mathrm{g}_{\mathrm{m}}$ of $1200 \mathrm{mS} / \mathrm{mm}$. These three stage amplifiers demonstrated a noise of $18 \mathrm{~K}$ at $40 \mathrm{GHz}$, with good bandwidth (Fig. 2). We have processed a large number of them in several foundry runs and they are currently operating in CAPMAP instrument [5]. Fig. 2 shows the photograph of the circuit with its measured cryogenic performance and the measured performance of a CAPMAP receiver amplifier module.
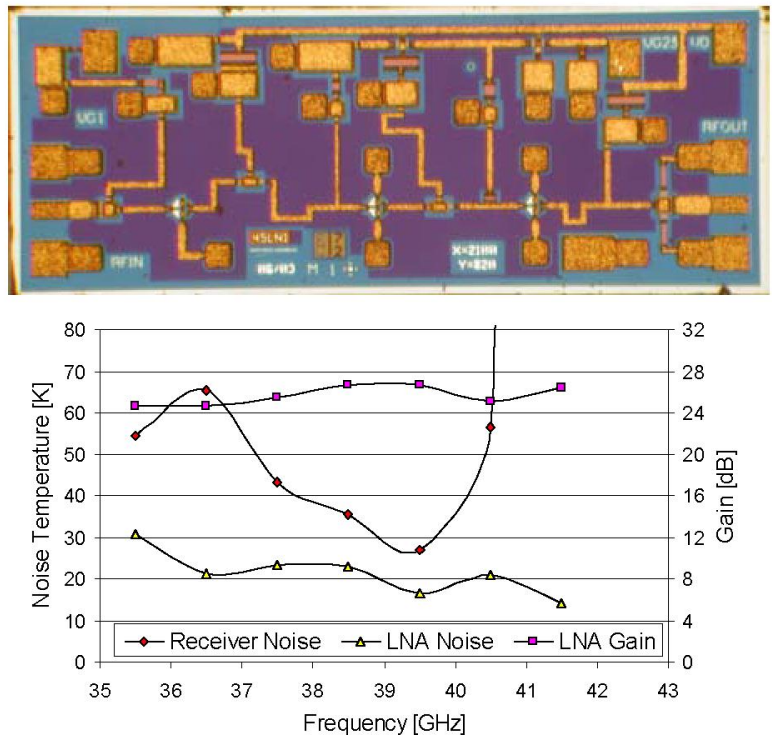

Fig. 2. The photograph of the three stage InP LNA MMIC (2.1 x $0.8 \mathrm{~mm}^{2}$ ) and the measured noise temperature at $20 \mathrm{~K}$ physical temperature. The graph includes the measured noise of a typical cryogenically cooled CAPMAP receiver amplifier module.

\section{B. Phase switch MMIC}

The 180 degree phase switch MMIC is based on the design developed for PLANCK Low Frequency Instrument [6]. The topology of the phase switch is two back-to-back rat race hybrids with an output low power SPDT switch (Fig 3.). The
SPDT switch is used to couple one of the hybrid outputs to the MMIC output and to create an open circuit to the other hybrid output. These phase switches were implemented on an HBT InP MMIC process that has PIN diodes. Fig 4. shows the low insertion loss of $2 \mathrm{~dB}$ and excellent magnitude $(+/-0.1 \mathrm{~dB})$ and phase $(+/-1$ degrees) balance over our operating bandwidth.

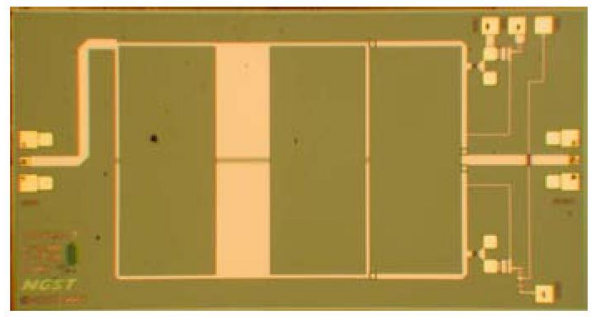

a)

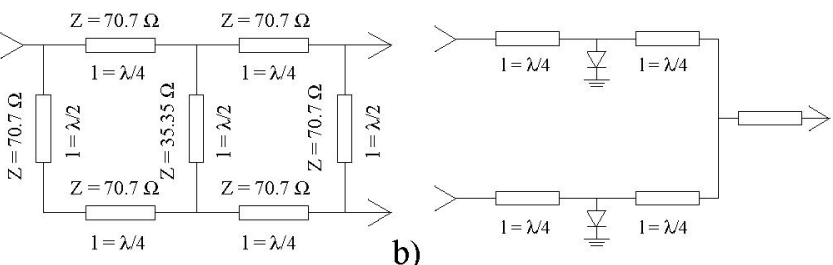

Fig 3. The photograph of the InP PIN MMIC phase switch $\left(2.43 \times 1.37 \mathrm{~mm}^{2}\right)$ and the schematic [6] of the a) 180 degree hybrid and b) low power SPDT switch. By cascading the 180 degree hybrid and the SPDT switch the MMIC phase switch is built.

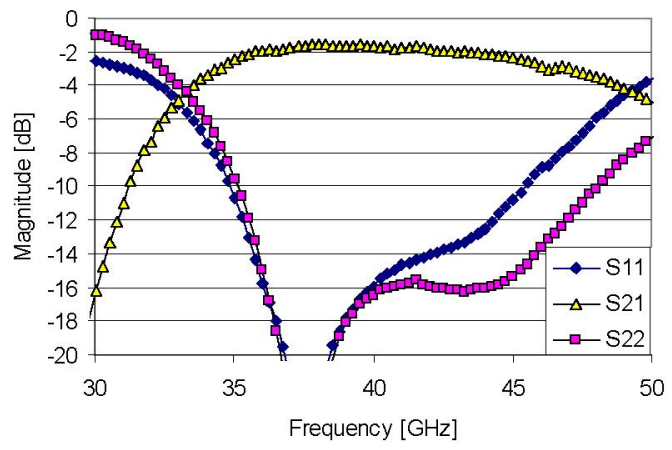

a)

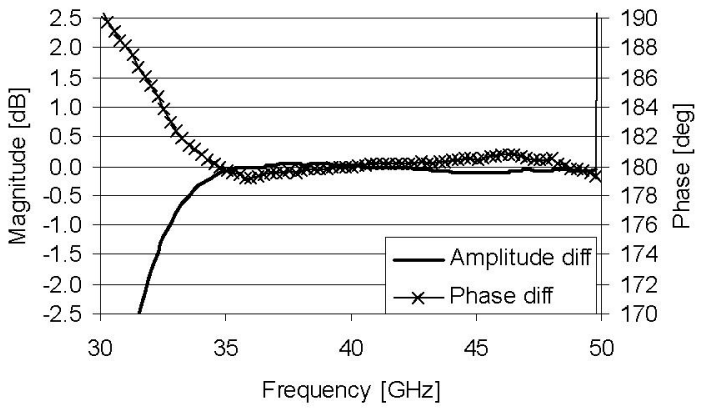

b)

Fig. 4. Measured a) response and return loss and b) amplitude and phase difference between the two states of the phase switch MMIC. 


\section{Polarization hybrid}

To detect the Stokes parameters, a planar phase discriminating hybrid was designed and characterized (Fig. 5). This hybrid was designed together with all the other passive circuitry (detector substrates, band pass filters, attenuators and interconnects) on 4 mil Alumina substrates using thin film technology. The hybrid combines the 180 degree hybrid, 90 degree hybrid and intermediate power splitters shown in Fig. 1 on a single alumina substrate. Planar 180 degree hybrids have been previously implemented by combining 90 degree hybrid and two 90 degree phase shifters [7].

The 180 degree hybrid was designed with broadband 90 degree phase shifters $[8]$ and broadband multisection branchline couplers [9]. To improve the match and bandwidth of the phase shifters we tuned the impedance of the section connecting the coupled lines together [10]. A further improvement in performance was achieved by placing the Wilkinson power splitters before the second phase shifter. It provided isolation between the 90 degree hybrid and the phase shifter without affecting the detection of the polarization parameters. The final 90 degree hybrid is again a multisection branchline coupler. Fig. 5 shows the hybrid and the measurement and simulation results for the four outputs.

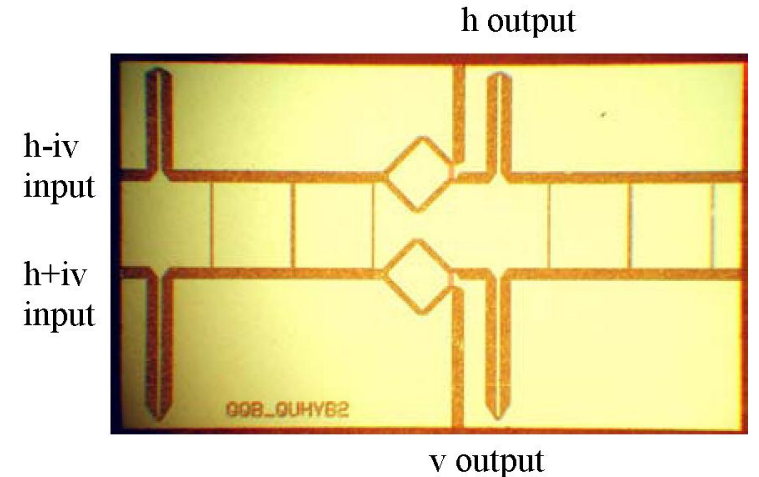

$\mathrm{h}+\mathrm{v}$ output

h-v output
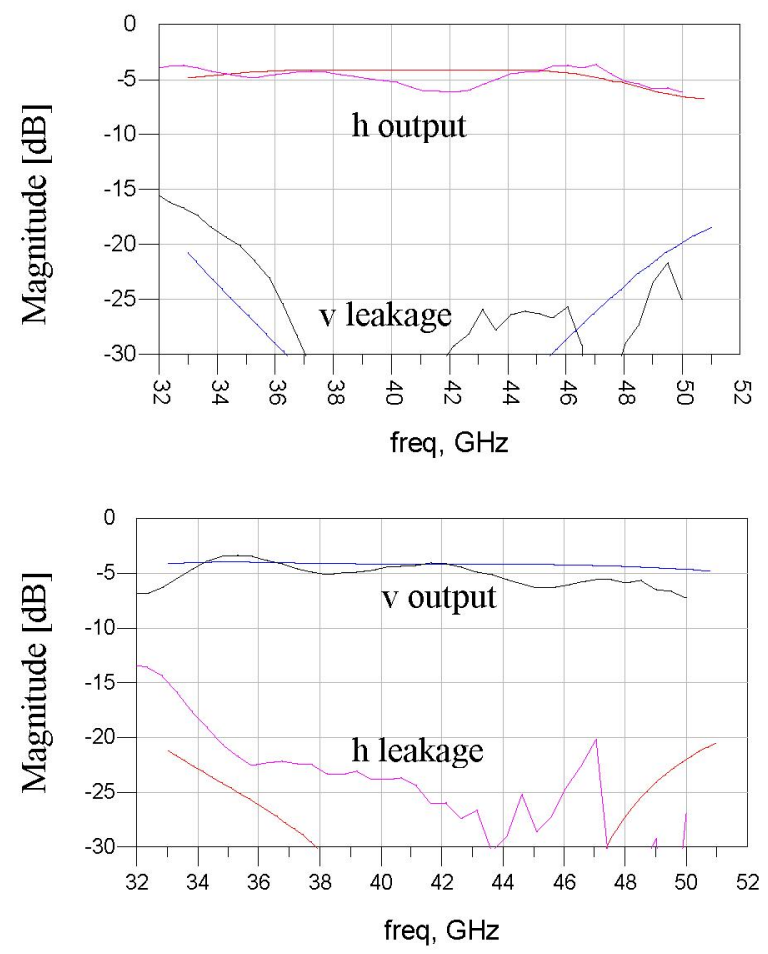
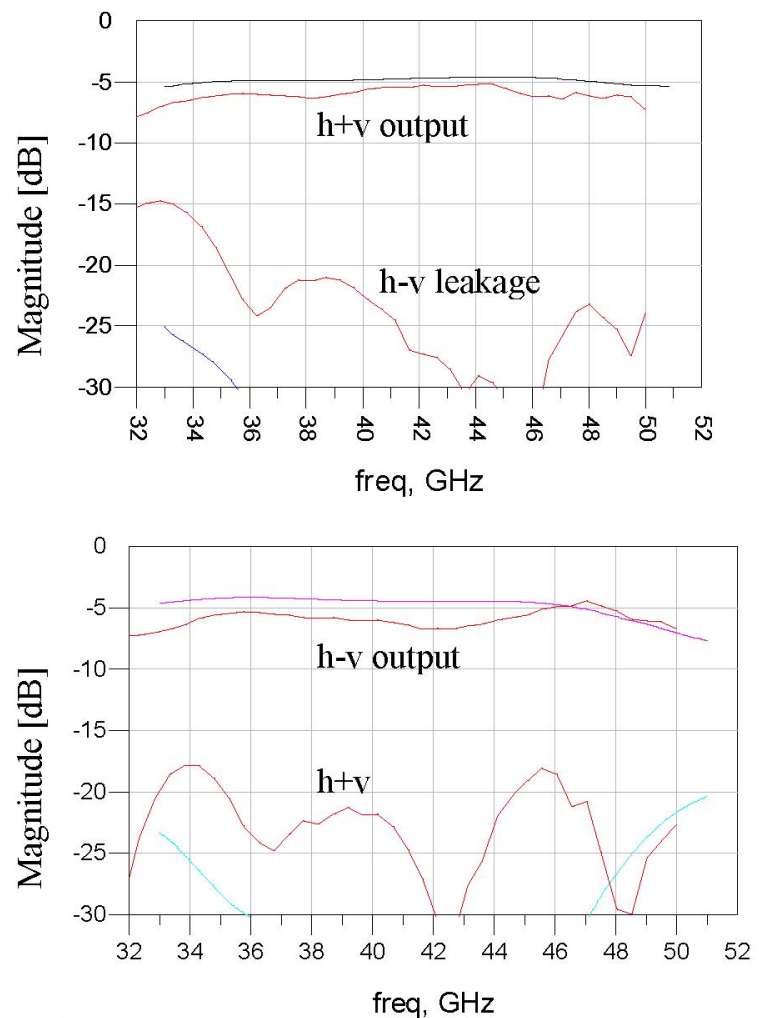

Fig. 5. Planar Q, U phase discriminator hybrid for the MCM. This hybrid enables the detection of the Stokes parameters $Q$ and $U$ in a planar module. The on-wafer measured and simulated outputs of the hybrid are shown.

\section{Planar Polarimeter DeVelopment}

The complete planar Q-band package that integrated all parts was designed to be 2"x 2" (Fig. 6). The modules had 34 pins that plugged into a simple printed circuit board for bias control and tuning provided through an automated computer interface, eliminating costly manual tune and test.

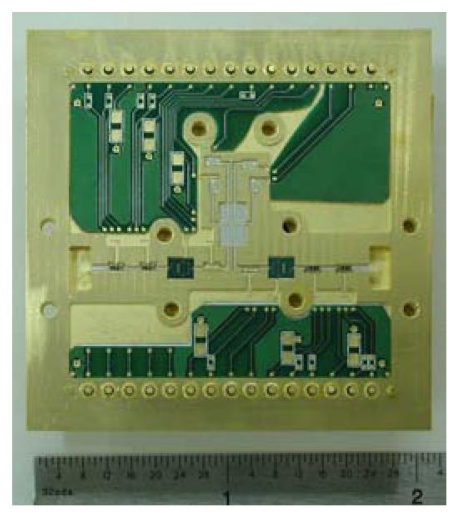

Fig. 6. $40 \mathrm{GHz}$ polarimeter integrated as a MultiChip Module (MCM). The module contains all the functions of the polarimeter, including the detection of Stokes parameters $\mathrm{Q}$ and $\mathrm{U}$. The module has two WR-22 waveguide inputs from the OMT through the lid and DC connections to the back-end electronics 
These modules were characterized in cryogenic measurements (Fig. 7) and achieved a noise temperature of less than $25 \mathrm{~K}$, which is close to the expected result based on the characterization of the amplifier (Fig. 2).
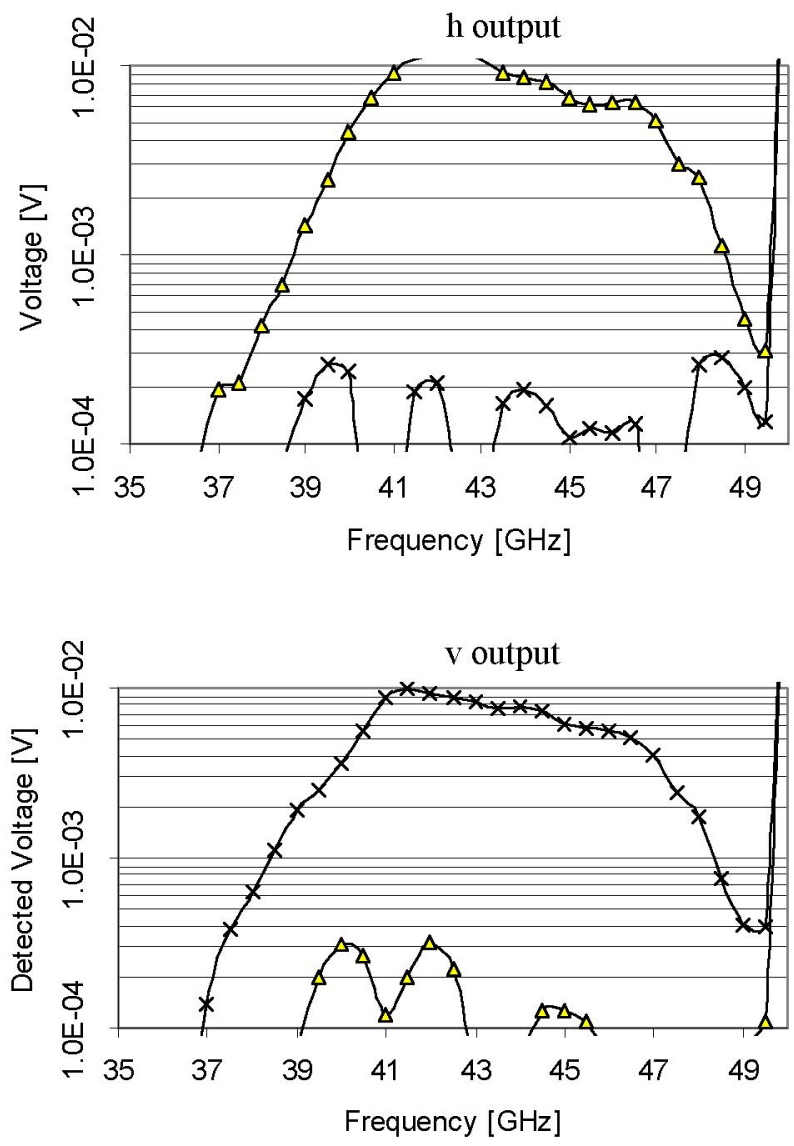

Fig. 7. Cryogenic measurement results of the $h$ and $v$ outputs of the module. These tests were measured with a magic- $T$ in front of the module and thus only the $h$ and $v$ outputs could be measured. We are currently testing the modules with circular OMT to characterize all four outputs of the module.

These modules form complete correlation polarimeters that are connected to the antenna, and supply detector outputs at DC. These multiple detectors provide the measurement of Stokes parameters Q and $\mathrm{U}$ simultaneously, which is not possible with direct detectors. There are no other cables or connectors in the modules, and the large numbers of pins in the plug-in modules allow independent optimization of bias for all amplifiers. The biasing and readout circuitry is all placed on standard PCBs. All of the parts can be mass produced inexpensively which enables scaling to thousands of elements.

\section{CONCLUSION}

We developed planar Q-band polarimeters that provide the Q and $\mathrm{U}$ Stokes parameters simultaneously. These planar polarimeters have a noise temperature of less than $25 \mathrm{~K}$ over 8 $\mathrm{GHz}$ bandwidth and are easily scalable to a large array. A massive array will provide vital information on foregrounds, systematics and polarization science. This breakthrough in millimeterwave packaging/mass production technology enables arbitrarily large arrays at modest cost. It is adaptable for polarimetry, intensity radiometry, and spectroscopy. It will thus revolutionize many areas of radio astronomy and Earth remote sensing. For CMB polarimetry, amplifier arrays offer the best technology for ground-based observations up to 100 GHz.

\section{ACKNOWLEDGEMENT}

The research described in this paper was carried out at the Jet Propulsion Laboratory, California Institute of Technology, under a contract with the National Aeronautics and Space Administration. The authors wish to acknowledge the support of Roger Hoyland in phase switch development and the support of the Q,U, Imaging ExperimenT collaboration.

\section{REFERENCES}

[1] E. M. Leitch, J. M. Kovac, C. Pryke, J. E. Carlstrom, N. W. Halverson, W. L. Holzapfel, M. Dragovan, B. Reddall, and E. S. Sandberg. "Measurement of polarization with the Degree Angular Scale Interferometer," Nature, Vol. 420, December 2002. pp. 763-771.

[2] Todd Gaier, Charles Lawrence, Michael Seiffert, Mary Wells, Pekka Kangaslahti, and Douglas Dawson, "Amplifier Arrays for CMB Polarization," CMB Polarization Workshop, University of Minnesota, Minneapolis, March, 2003.

[3] Michael Seiffert, "QUIET Array Overview," QUIET Polarization Meeting, Princeton University, Princeton, NJ, September, 2003.

[4] http://quiet.uchicago.edu

[5] http://wwwphy.princeton.edu/cosmology/capmap/

[6] R. Hoyland, "A New MMIC, Wideband $180^{\circ}$ Phase Switch Design for Millimeter Wave Applications," Proc. of the $3^{\text {rd }}$ ESA Workshop on Millimeter Wave Technology and Applications, MilliLab, Espoo, Finland, 21-23 May, 2003. pp. 305-310.

[7] D. I. Kraker, "Asymmetric Coupled-Transmission-Line MagicT," IEEE Transactions on Microwave Theory and Techniques, vol. 12, pp. 595 - 599, November 1964.

[8] B. M. Schiffman, "A New Class of Broad-Band Microwave 90Degree Phase Shifters," IEEE Transactions on Microwave Theory and Techniques, vol. 6, pp. 232 - 237, April 1958.

[9] S. Kumar, and T. Danshin, "Multisection Broadband Impedance Transforming Branchline Quad Hybrid Suitable for MMIC Realisation," Electronics Letters, vol. 28, pp. 1368-1370, July 1992.

[10] Y. C. Leong, and S. Weinreb, "Novel technique of phase velocity equalization of microstrip coupled-line phase shifters," 2000 IEEE MTT-S Int. Microwave Symp. Dig., vol. 3, pp. 1453 - 1456, June 2000. 